\title{
DETECTION OF STRONG WINDS IN GIANT EXTRAGALACTIC HII REGIONS
}

\author{
H.O. CASTAÑEDA and J.M. VILCHEZ \\ Instituto de Astrofísica de Canarias \\ E-38200, La Laguna, Tenerife, Spain
}

\begin{abstract}
The existence of supernova remnants and strong stellar winds in giant extragalactic HII regions should be associated with the existence of gas at very high velocities $\left(\approx 10^{2}-10^{3} \mathrm{~km}\right.$ $\mathrm{s}^{-1}$ ). The detection of large velocity/low intensity gas within the regions could be possible with the use of CCD detectors with long slit intermediate spectroscopy and long integration times. To test this observational approach we have conducted a survey in the brightest giant HII regions of M 101 and M 51. We report on the preliminary results of our programme, and discuss the implications for the energetics of the regions.
\end{abstract}

K. A. van der Hucht and B. Hidayat (eds.),

Wolf-Rayet Stars and Interrelations with Other Massive Stars in Galaxies, 423.

(C) 1991 IAU. Printed in the Netherlands. 